\title{
PRÁCTICAS PRODUCTIVAS DE LOS SISTEMAS FAMILIARES DE CRÍA CAPRINA EN LOS VALLES CALCHAQUIES (PAYOGASTA, SALTA)
}

\author{
SuÁreZ, V. H. ${ }^{1}$; Martínez, G. M. ${ }^{2} ;$ Olmos, L. H. ${ }^{1} \&$ Arapa, C. ${ }^{3}$
}

\section{RESUMEN}

Una encuesta transversal se llevó a cabo en 35 unidades productivas familiares (UPF) que criaban caprinos en el Municipio de Payogasta (Salta) con el objetivo de describir las prácticas productivas. Se recabaron datos del manejo, alimentación, reproducción y sanidad de los caprinos. Las UPF criaban en promedio 80,1 $\pm 57,1$ caprinos (sin contar cabritos). El biotipo predominante fue Anglo Nubian cruzados con Criollo. El sistema productivo fue extensivo con encierre nocturno y la alimentación se basaba en el pastoreo de los cerros, de alfalfa, rastrojos o residuos de cosecha. El $100 \%$ de las UPF practicaba el ordeñe manual destinado a elaborar quesos. En el 51,4\% de las UPF el servicio era estacionado, mientras que en el resto continuo. En el 65,7 \% de las UPF se desparasitaba rutinariamente. Aquellas UPF que estacionaban servicio y desparasitaban mostraron una tendencia a obtener mejores valores productivos. Estos resultados muestran limitantes productivas que con prácticas sencillas sería posible incrementar la producción y elevar el bienestar de las familias.

Key words: encuesta, caprinos, prácticas productivas, agricultura familiar, Valles Calchaquíes.

\begin{abstract}
Productive practices of small-holder goat flocks in the Calchaquies Valleys (Payogasta, Salta).

A cross-sectional survey was performed in 35 family farming units (FFU) in the Municipality of Payogasta (Salta), with the aim of describing production practices in their goat flocks. Data on management, feeding, reproduction and health practices were recorded. FFU had on average $80.1 \pm 57.1$ goats (not including kids). The predominant biotypes was Anglo Nubian (60.5\%) with
\end{abstract}

1.- INTA, Área de Investigación en Salud Animal- IIACS-CIAP con sede en EEA Salta, Argentina. Email: suarez.victor@inta.gob.ar

2.- INTA EEA Salta, Ruta Nacional 68 km 172. (4403) Cerrillos, Salta, Argentina.

3.- Municipalidad de Payogasta, Salta, Argentina.

Manuscrito recibido el 11 de diciembre de 2019 y aceptado para su publicación el 26 de febrero de 2020.

Suárez, V.H.; Martínez G.M.; Olmos, L.H. \& Arapa, C. Prácticas productivas de los sistemas familiares de cría caprina en los Valles Calchaquies (Payogasta, Salta). FAVE - Ciencias Agrarias 19 (1): 97-110. CC BY-NC-SA 4.0 (c) (i) (2) 


\section{H. Suárez et al.}

Criollo crosses. Animals are extensively managed, with nocturnal enclosure, and flock feeding was based on grazing the hills, lucerne, crops or their residues. The $100 \%$ of the FFU produced milk by hand-milking to make cheeses. Breeding was stationed (51.4\%), while in the rest it was continuous. The $65.7 \%$ of the FFU routinely dewormed. Those FFU that had stationed mating system and dewormed showed to obtain a tendency of higher production values. These results show important productive limitations that, with simple practices it would be possible to increase production and the well-being of producing families.

Key words: survey, goats, production practices, family farming, Calchaquies Valleys.

\section{INTRODUCCION}

Dentro del Departamento de Cachi, ubicado en los Valles Calchaquíes, el territorio correspondiente al municipio de Payogasta se caracteriza por su extrema aridez, un relieve montañoso dominado por los nevados de Cachi $(6380 \mathrm{~m})$ y de Palermo (6120 m) donde predominan serranías y quebradas por donde bajan los cursos de agua fundamentales para el riego de la producción agrícola y en menor medida para la cría del ganado. Estas actividades constituyen la principal fuente de ingresos de la población, compuesta casi en su totalidad por productores minifundistas propietarios de pequeños predios o en muchos casos arrendatarios u ocupantes de tierras fiscales. Los principales productos de estas economías familiares de acuerdo a las posibilidades de su ubicación y acceso al agua son pimiento para pimentón y en menor medida alubias, tomate, comino, alfalfa, arvejas, maíz y la cría mayormente de pequeños rumiantes (Manzanal, 1987). Esta última actividad se basa en la cría de cabras y en menor medida ovejas, siendo los quesos artesanales, los cabritos, corderos y capones los principales productos para el autoconsumo o para la venta, principalmente informal de los excedentes en las poblaciones cercanas.
Este tipo de cría extensiva de caprinos y de producción estacional tiene graves condicionantes que afectan la competitividad de estos sistemas familiares, que aspiran a incorporar sus productos como queso y carne a la creciente demanda de la actividad turística de la región. Entre esas limitantes podemos citar la escasa adopción de tecnologías simples en el manejo alimenticio, genético, reproductivo y sanitario de las majadas, como también medidas que garanticen la inocuidad y calidad de los productos pecuarios (Martínez y Suarez, 2019).

En lo referente a esta problemática de la producción caprina a nivel de la agricultura familiar o de minifundios existen, aunque no numerosas, encuestas y estudios en diferentes regiones del país (Dayenoff y Carrizzo, 1993; Rigalt et al., 1993; Gutman et al., 2004; Paz et al., 2005; Bedotti et al., 2007; Chavez et al., 2011; Suarez et al, 2014b; 2015). Pero la información sobre aspectos relacionados con el manejo de las majadas, su alimentación, reproducción o su genética en la región del NOA son aún más escasos y faltan estudios que posibiliten el desarrollo de estrategias apropiadas a estos sistemas de producción familiar.

A partir de estas carencias, el objetivo de la presente encuesta llevada a cabo entre los productores de caprinos del Muni- 
cipio de Payogasta, fue caracterizar prácticas relacionadas con el manejo forrajero y alimenticio, reproductivo, sanitario con el propósito de estar en condiciones de poder proponer y transferir tecnologías que permitan lograr mayor competitividad a esta producción tan necesaria para las familias de esta región representativa de los Valles Calchaquíes de Salta.

\section{MATERIALES Y MÉTODOS}

\section{Tipo y lugar de la encuesta}

Una encuesta transversal se llevó a cabo entre junio y octubre del 2018 en 35 unidades productivas familiares (UPF) que criaban caprinos ubicadas en el Municipio de Payogasta, departamento de Cachi, provincia de Salta (Figura 1). Las UPF se ubicaron en los siguientes parajes: Buena Vista $(n=8)$, Payogasta $(n=7)$, Palermo $(n=6)$, Punta de Agua $(n=4)$, Tonco $(n=3)$, Belgrano ( $n=3)$, Piul $(n=3)$, Arqualito $(n=1)$. El área de estudio está inserta en los denominados Valles Calchaquíes, región donde

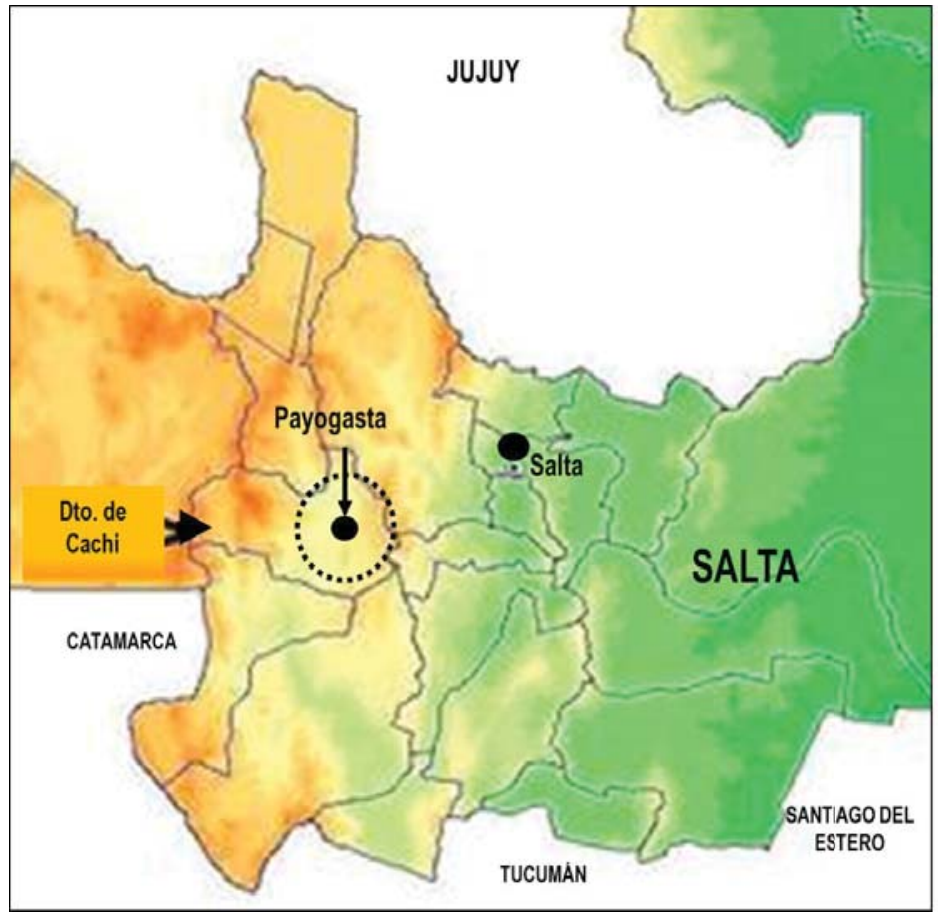

Figura 1: Ubicación de la región del Municipio de Payogasta (Dto de Cachi, Salta) en donde se llevó a cabo la encuesta a las familias criadoras de caprinos. 
a sus habitantes los une una remota tradición cultural y una geografía que comparte montañas, valles y quebradas, de extrema aridez con precipitaciones promedio de alrededor de $200 \mathrm{~mm}$ ubicadas entre fin de diciembre y marzo.

La encuesta se realizó por conveniencia, seleccionando aquellas UPF representativas de la región de acuerdo a la tenencia de cabras según referentes del Municipio de Payogasta, que estaban dispuestas a recibir en su predio la visita de los encuestadores.

\section{Cuestionario}

A partir de la respuesta de los productores de cada UPF se completó un cuestionario que constó de una lista de 60 preguntas. La encuesta se constituyó de una parte inicial donde se recabaron datos generales de la explotación tales como número de caprinos, categorías, hectáreas propias, actividad principal, infraestructura, entre otros y de otra parte referida a prácticas utilizadas en el manejo forrajero y alimenticio, el reproductivo, sobre la genética, acerca del manejo sanitario y preguntas generales de índices productivos y aspectos de comercialización. En cada una de las 35 UPF se observaron los animales y las instalaciones.

\section{Análisis de los datos}

Los datos fueron chequeados a diferentes niveles para descartar errores y en algunos casos de inconsistencias se volvió a llamar telefónicamente al productor a través del Municipio. Para el análisis estadístico de las variables se utilizaron en primera instancia, técnicas descriptivas (Thrusfield, 1997), el análisis de varianza no paramétrica de Kruskal Wallis debido a la falta de normalidad en la distribución de los datos y se calculó el odds ratio (OR).

\section{RESULTADOS Y DISCUSIÓN}

\section{Características de las UPF}

Los productores encuestados eran propietarios (48,6\%) o arrendatarios (34,3\%) de pequeñas superficies dedicadas principalmente a la agricultura (promedio: 3,1 $\pm 2,5 \mathrm{ha}$ ) o puesteros sin propiedad (17,1\%), criando la mayoría sus animales durante gran parte del año sobre tierras fiscales, sin alambrados, ni límites definidos. Las explotaciones y existencias totales del departamento de Cachi de acuerdo a los datos del RIAN (2010) se estiman en 52 UPF y 2778 caprinos y según estos datos el porcentaje de las UPF encuestadas fue de 67,3\%. Cabe señalar que hasta el presente Salta no posee un relevamiento provincial que actualice la existencia de cabezas caprinas en la provincia. Esta carencia de cifras certeras sería avalada por las presentes observaciones, ya que si sumamos las existencias caprinas de solo las 35 UPF encuestadas nos da un total de 2803 cabezas sin considerar cabritos menores de 3 meses, demostrando que las cifras oficiales subestiman extensamente la cantidad real de cabezas de todo el Dto. de Cachi.

Las UPF muestreadas criaban en promedio 80,1 $\pm 57,1$ caprinos, sin contar los cabritos menores a los 45 días de edad (44,7 $\pm 41,6$ ) debido a que su número al momento de las visitas variaba de acuerdo si estaban en plena parición o si habían sido consumidos o vendidos. La figura 2 muestra los promedios de las existencias caprinas por categorías que criaban las UPF.

En cuanto a la composición genética de las majadas, en el caso de los reproductores cabríos, también denominados "hechores", el 68,3\% era de raza Anglo Nubian o de cruzas de ésta con biotipos de raza Criolla; un 20,0\% de biotipo Saanen cruzado 


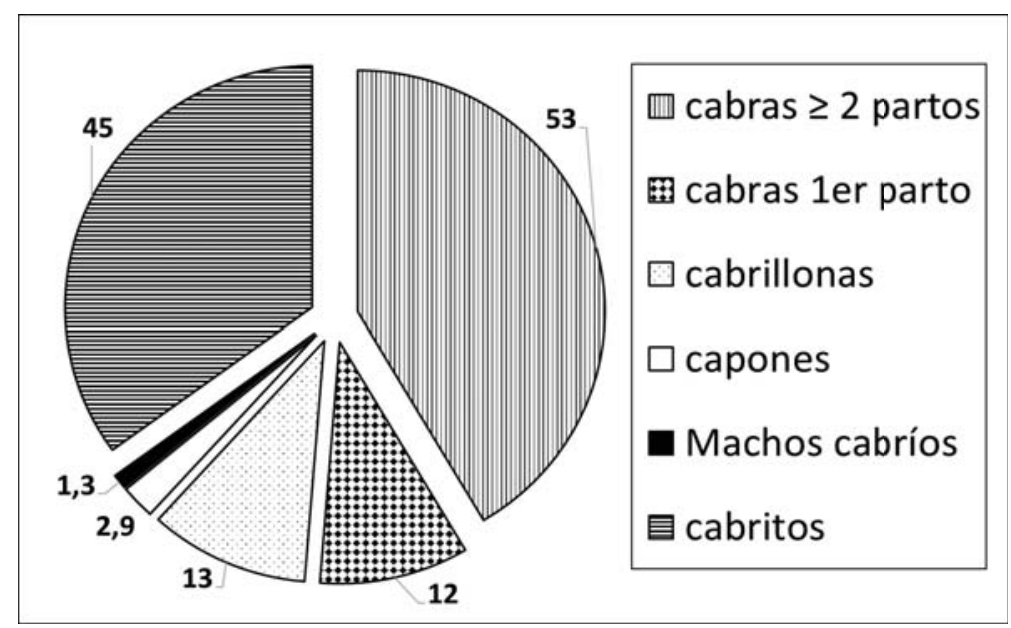

Figura 2: Promedios de las existencias caprinas discriminadas por categorías registradas a partir de las unidades productivas familiares.

con Criollo y un $11,7 \%$ con características cercanas al biotipo Criollo. La genética de las hembras respondió en un 60,5\% al biotipo Anglo Nubian cruzado con Criollo; el $18,6 \%$ Criollo con sangre Saanen u otra; un $20,9 \%$ a las características del Criollo. Como punto a considerar se debe decir que, aunque la denominación Criollo corresponde a caprinos traídos por los conquistadores que luego de un proceso de selección natural se constituyó en una raza con particularidades ya propias, adaptada a la región (Poli et al., 2005), la elevada introducción de reproductores Anglo Nubian o Saanen dificultan precisar a partir de los fenotipos observados que cabras respondían a las características descriptas para la raza Criollas de tipo andino (Fernández et al., 2014).

En cuanto a la cría de otras especies el $82,4 \%$ de las UPF criaba ovinos, el 20,6\% bovinos y el $41,2 \%$ cerdos. Además, un $79,4 \%, 100 \%$ y $76,5 \%$ de las UPF declararon tener respectivamente equinos, perros y gatos; el $100 \%$ de las familias criaban tam- bién gallinas y algunas otras aves de corral. A diferencia de lo observado en la región del Chaco semiárido salteño (Suarez et al, 2015), donde el $31,6 \%$ de las UPF se caracterizaron por criar bovinos, la falta de cría bovina en esta región probablemente se deba a las características productivas de estos sistemas mayormente agrícolas y por otro lado a la aridez y escases de pasturas de la región.

Las instalaciones de las UPF fueron precarias, en su mayoría solo constaban de no más de 2 corrales (uno de verano y otro de invierno) de alambre romboidal, generalmente provistos de chapas a los laterales como reparo del viento; en los parajes más alejados también se observaron corrales de piedra. Algunos corrales tenían sombra y como subdivisión, un lugar especial para los cabritos que por lo general constaba de un techo de chapa y reparo en los laterales. Los comederos y bebederos cuando existían estaban hechos a partir de cubiertas de vehículo o de recipientes plásticos cortados por la mitad. 


\section{H. Suárez et al.}

En cuanto a su higiene, al cambiar de corral una a dos veces al año se retiraba el guano de las cabras, que era utilizado para la fertilización de los sembradíos, huertas o frutales.

A pesar de ser prioritario para la agricultura, el recurso agua de bebida para la majada en general se declaró como suficiente y de buena calidad $(91,4 \%)$ y suministrada a partir de vertientes o de las acequias.

\section{Prácticas de Manejo}

Las mujeres en general dirigían el manejo de la majada y conocían bien a sus cabras, siendo en un 44,1\% tarea exclusiva de ellas o en un 38,2\% acompañada por toda la familia; solo en un 17,6\% la majada estaba a cargo en forma exclusiva por hombres a pesar de ser la agricultura su principal responsabilidad, probablemente debido a la falta de cría ganado bovino que por lo general es la ocupación masculina por excelencia en otras regiones (Suarez et al., 2015).

En cuanto al manejo forrajero de la majada, el 65,9\% de los productores la llevaban a pastar a los cerros y vegas (siempre con el cuidado de un pastor) a partir de la existencia de buena disponibilidad de pasto desde fines de diciembre-enero hasta abril-mayo, que es cuando regresan las cabras a parir cerca de las casas; el resto de las UPF (34,1\%) durante este período no practica el pastoreo de los cerros y alimenta a la majada todo el año cerca de sus viviendas a base a alfalfa y pasto natural. A partir de abril mayo cuando por lo general comienza la parición, la mayoría de las UPF (70,4\%) alimenta la majada a base a los rastrojos agrícolas (pimiento, tomate, arveja, maíz) complementados con heno de alfalfa o chala de maíz, a su vez el 29,6\% restante continúa con el pastoreo de alfalfa hasta que los fríos del invierno los obligan a complementarla con rastrojos, grano, algarrobas, etc. Entrada la primavera casi el 75\% de las UPF utilizan como alimento principal el pastoreo de la alfalfa complementado con rastrojos o las reservas forrajeras que les quedan disponible a la espera de poder llevar de nuevo las majadas a los cerros. La figura 3 ejemplifica a lo largo de las estaciones del

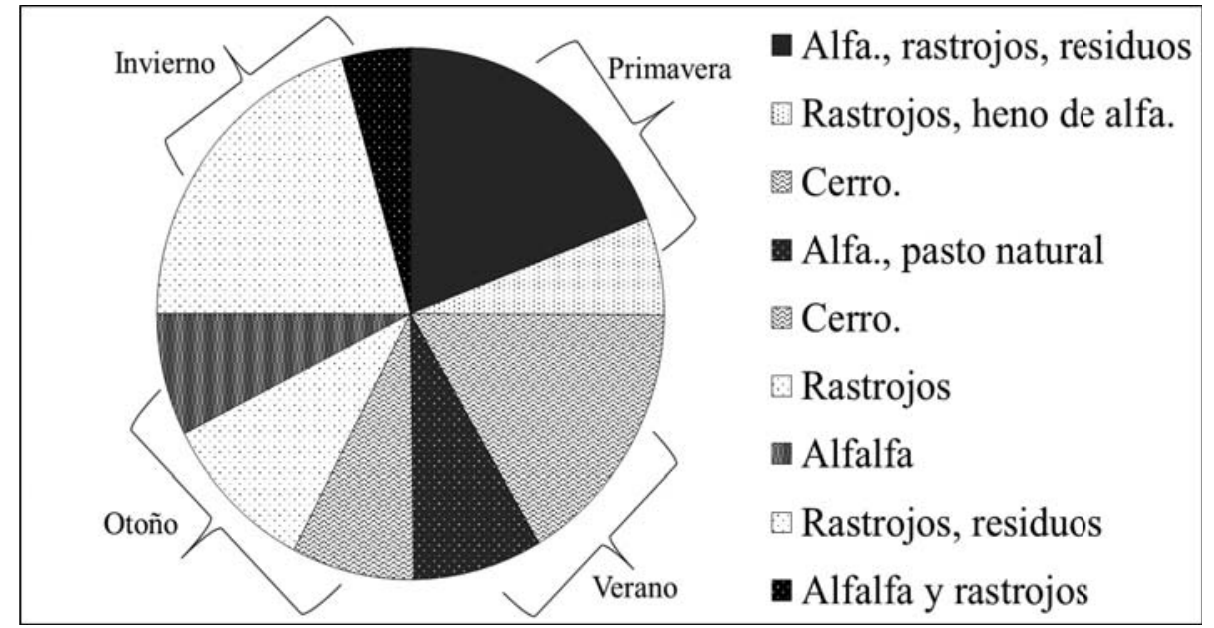

Figura 3: Porcentaje de los principales recursos forrajeros ofrecidos a las majadas caprinas a lo largo de las estaciones del año por las unidades productoras familiares encuestadas. 
año los principales recursos forrajeros explotados. El manejo forrajero muestra diferencias entre los parajes: aquellos cercanos a los pueblos de Payogasta y Cachi tiene pocas chances de utilizar el pastoreo de los cerros a diferencia de los que están más alejados o aquellos parajes con mejor disponibilidad de riego como Palermo utilizan mayormente a la alfalfa como base forrajera, aunque cabe aclarar que la disponibilidad de los alfalfares dista mucho de ser la ideal.

En el $80 \%$ de las UPF se suplementa solo a las recién paridas, a los cabritos mamones o a las cabras en ordeño con unos 50 a 200 g maíz, heno de alfalfa, algarrobas y suministra sales en panes para lamer.

En general el aprovechamiento del pastoreo en los cerros o de áreas comunes más alejadas de las viviendas, aunque económicamente es más rentable, arroja datos productivos que tienden a mostrar menores tasas productivas, como se señala en la tabla 1. Probablemente, esta tendencia se deba a la imposibilidad de brindarle a la majada un mayor cuidado y cubrir mejor sus demandas energéticas. En un mismo sentido, en las quebradas de Jujuy y Salta las UPF con un manejo sin o con poca permanencia de pastoreo en los cerros se observó una mayor producción de leche en comparación con aquellas UPF que permanecían con sus cabras más tiempo en los cerros (Suarez et al., 2017a).

La cría fue extensiva con encierre nocturno y donde aproximadamente en un $70 \%$ de las UPF, las cabras luego de ser ordeñadas entre las 9 y 11 h de la mañana salen a pastorear a partir de las 14 hasta las 18:30 h, cuando vuelven al corral. Esta rutina se observó en otras regiones como en las UPF de las quebradas de Humahuaca y del Toro (Suarez et al., 2017a). Por otro lado, algunas UPF envían sus cabras a pastoreo dos veces al día, entre 10 y 13 h y entre 16 y 18:30 h.

El sistema de ordeñe se presenta básicamente de dos formas: a) se ordeña sin cabrito al pie (53,1\%) desde los 2 a 3 meses de edad cuando alcanza los 7 a $10 \mathrm{~kg}$ y o es vendido; b) a media leche (46,9\%) desde los 40 a los 85 días de vida del cabrito y dentro de esta última forma un $9,4 \%$ ordeña día por medio para no perjudicar la ganancia de peso de los cabritos. Los cabritos por lo general quedan en el corral y solo maman luego del ordeño, antes de liberar sus madres a pastoreo o cuando tienen más edad maman durante el pastoreo. Por la noche los cabritos son separados o se les colocaba un morral para que no mamen de sus madres.

Tabla 1: Producción de leche individual y total por lactancia por cabra, porcentaje de cabritos señalados sobre cabras servidas y tasa de mortalidad perinatal, de acuerdo a si utilizan el pastoreo de los cerros o no.

\begin{tabular}{|c|c|c|c|c|}
\hline $\begin{array}{l}\text { Aprovecha el } \\
\text { cerro }\end{array}$ & $\begin{array}{l}\text { Leche/diaria } \\
\text { (litros) }\end{array}$ & $\begin{array}{l}\text { Leche total } \\
\text { (litros) }\end{array}$ & $\begin{array}{l}\text { Cabritos señalados } \\
\text { /Cabras servidas }\end{array}$ & $\begin{array}{l}\text { Mortalidad } \\
\text { Perinatal }\end{array}$ \\
\hline Si $(n=18)$ & 0,60 & 95,9 & $75,7 \%$ & $13,9 \%$ \\
\hline No $(n=15)$ & 0,71 & 117,9 & $85,0 \%$ & $17,0 \%$ \\
\hline
\end{tabular}




\section{H. Suárez et al.}

Por lo general el suplemento de los animales está lejos de cubrir sus necesidades alimenticias, las cuales también se verían afectadas debido al poco tiempo de que disponen las cabras para alimentarse en pastoreo (entre 4 y 6 h) mientras están bajo ordeño. Una razón de este manejo es que la familia y especialmente las mujeres en algunas épocas del año no disponen de tiempo debido a otras responsabilidades familiares ni tampoco ayuda para soltar y cuidar la majada luego del ordeñe, ya que deben elaborar el queso inmediatamente.

\section{Tipo de ordeño y comercialización de productos}

En el $100 \%$ de las UPF se ordeña manualmente y en el corral. El número promedio de cabras que se ordeñan fue de 29,2 \pm 14 , variando de acuerdo la secuencia de pariciones. La producción promedio por cabra se estimó en 0,65 $\pm 0,3$ litros durante 2,5 a 6 meses, con una producción total promedio de 102,5 \pm 58 litros al año por cabra de acuerdo al tipo de ordeño o edad de destete del cabrito. Estos datos guardan semejanza con otras áreas de clima similar como son las quebradas áridas (Suarez et al., 2017a). Entre productores se observaron diferencias notables, entre los que se acercaban al litro por cabra diario y otros que no superaban los $300 \mathrm{ml}$ diarios; estas diferencias se podrían atribuir a la dieta basada en la alfalfa manejada por algunas UPF en contraste con otras que solo disponían de rastrojos de cosecha. Aunque datos productivos controlados de las majadas de productores chicos en condiciones de explotación extensivas son muy escasas, en Santiago del Estero bajo mejores condiciones de explotación y principalmente con biotipos cruza Anglo Nubian, se pudo registrar una variación en el rinde de las majadas de entre 34 a 173 litros totales (Paz et al., 2007; Fernández et al., 2007).

Cabe destacar la importancia que los productores le otorgan a la producción láctea por sobre la venta de cabritos, basada en aspectos tradicionales y de rentabilidad incrementada por el turismo, que marca una diferencia la región del Chaco semiárido salteño, donde solo en un $43,2 \%$ de las UPF se ordeña un porcentaje bajo de cabras luego de la venta de sus cabritos (Suarez et al., 2015).

En cuanto al rinde de la leche, los productores manifestaron obtener $1 \mathrm{~kg}$ de queso con 7,5 $\pm 2,0$ litros de leche. Estos datos están alineados con los elevados porcentajes de sólidos totales $(15,09 \pm 1,18 \%)$ hallados en estudios previos en los Valles Calchaquíes (Chávez et al., 2011). Aunque la leche es utilizada para elaborar el queso, esporádicamente en un 51,1\% de las UPF también se consume leche. El 92\% de los productores destinan la mayor parte del queso a la venta y al autoconsumo de los excedentes, y el 8 $\%$ restante solo al autoconsumo. Sólo en un 6,3\% de las UPF se ordeñaban también en escaso número ovejas y o alguna vaca para realizar queso mezcla.

Para la elaboración de los quesos los productores filtraban la leche, pero sin pasteurizarla y salaban en la cuajada usando para moldearlos ya sea el cinchón (66,7\%) o moldes plásticos (33,3\%). La venta era informal ya sea en forma directa en Cachi o Payogasta o mediante intermediarios que colocaban los quesos en la ciudad Salta y sus alrededores.

En cuanto a la comercialización de carne caprina, un 86,2 \% de las UPF venden del 50 al 75\% de su producción, mayormente cabritos de 2 a 3 meses de edad (7 a $10 \mathrm{~kg}$ de peso vivo) y en menor medida capones de más de 7 meses de edad o cabras adultas. El resto de la producción (cabras adultas o 
capones) es destinada al consumo propio. Estas ventas se realizan de manera informal principalmente a restaurantes de Cachi y en ocasiones a parientes, amigos, etc. También los cueros son otra fuente menor de ingresos. La producción de leche y la venta de queso como actividad principal de estos sistemas, difiere de la producción familiar de otras regiones del país, conocidas tradicionalmente como productoras de cabritos como por ejemplo en el oeste pampeano y Malargüe, la región cuyana y La Rioja, la región chaqueña, Santiago del Estero (Dayenoff y Carrizzo, 1993; Bedotti et al., 2007; Suarez et al., 2015; Contreras et al., 2016).

\section{Datos reproductivos}

Un 51,4\% de los productores encuestados estacionan el servicio mientras que el resto dejan los machos cabríos o hechores todo el año con la majada. De los productores que estacionan el servicio el 41,2\% alquila temporariamente los machos, mientras que el resto fuera de la época de servi- cio los tiene atados, maneados, encerrados o con delantales. De acuerdo a lo declarado por los productores el promedio del porcentaje de machos utilizados en servicio fue de $4,04 \pm 3,1 \%$. En cuanto a los períodos en los cuales estacionan servicio y las fechas de parición consecuentes se indican la figura 4. Cabe destacar el elevado porcentaje de productores que estacionan el servicio, a diferencia de otras regiones donde solo de un 3 o $4 \%$ a un $36 \%$ estaciona el servicio y donde no hay costumbre de alquilar los machos (Suarez et al., 2015; Suarez et al., 2017a; 2017b).

También en la figura 4 se observa la fecha natural de servicio y de partos de aquellos que no estacionan servicio, demostrando que la mayor parte de las cabras tienen celo y se sirven de diciembre a marzo, para luego ir disminuyendo la actividad sexual hacia el invierno. En muchos casos durante el pastoreo en los cerros los machos pueden servir las cabras de otras majadas debido a la falta de alambrados y originar partos

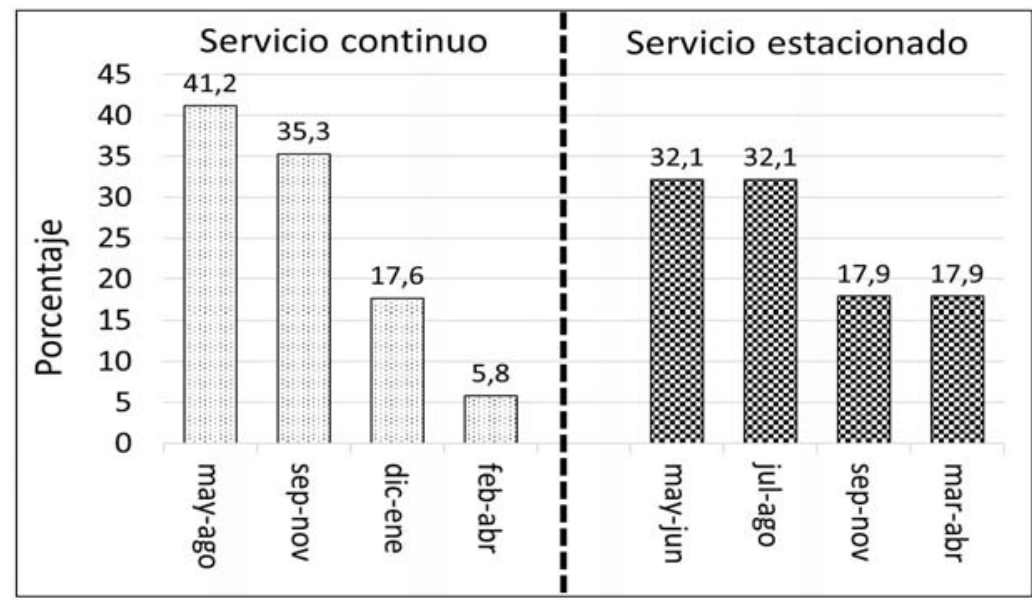

Figura 4: Porcentaje de las principales fechas de parición de las majadas caprinas declaradas por los dueños de las unidades productivas familiares de acuerdo al tipo de servicio: continuo o estacionado. 


\section{H. Suárez et al.}

en épocas no del todo deseadas. Si tomamos los períodos de parición de todas las UPF, la parición promedio se concentra en tres períodos, en abril-julio $(77,1 \%)$, septiembre-noviembre $(11,4 \%)$ y diciembre febrero (11,4\%). y una segunda parición de algunas cabras ya paridas en otoño que se reparte a su vez entre septiembre a noviembre $(50,0 \%)$ y diciembre a febrero $(31,8 \%)$. Esto muestra la alta presencia de celo desde el inicio al final del verano y una disminución de este hacia la primavera.

La tabla 2 muestra datos productivos entre aquellos que estacionan el servicio y los que no. El estacionamiento del servicio señala una tendencia a obtener valores productivos más elevados, con diferencias en lo que hace a la tasa $(\mathrm{p}<0,03)$ de mortalidad perinatal y a la producción $(\mathrm{p}<0,064)$ de leche total por cabra al año. Se halló una probabilidad 2,31 veces mayor de obtener más de $100 \mathrm{l}$ de leche total por cabra estacionando el servicio $(\mathrm{OR}=2,31)$. Trabajos previos en similares regiones áridas también demostraron que el estacionamiento del servicio también mejoró la señalada y disminuyó la mortalidad perinatal (Suarez et al., 2017a).

La estimación de los datos referidos a la tasa de mortalidad perinatal a partir del relato de los productores muestra un promedio general del $14,2 \pm 10 \%$, valor que se ve incrementado en las UPF que no estacionan servicio (Tabla 2). Estas cifras explicarían en parte las bajas tasas de cabritos señalados sobre cabras servidas. Por otro lado, este índice reproductivo sería aún menor si no se vieran compensados debido a que algunas cabras vuelven a presentar celo y otras logran tener dos pariciones anuales. Esta eficiencia de algunas cabras que paren dos veces en el año se observó en mayor porcentaje en la región chaqueña semiárida (Suarez et al., 2015). No fue posible precisar las causas de mortalidad perinatal, aunque gran parte de los productores declararon que una de las causas era la mortalidad por el frío y el viento y en menor medida las lluvias. Debido a que la mayoría de las cabras parían en el corral las muerte perinatal por predación (pumas, zorros) fue considerada como de poca importancia, salvo en aquellas UPF que no estacionaban servicio y un porcentaje de cabras parían anticipadamente en los cerros.

\section{Prácticas sanitarias}

Ningún productor declaró usar vacunas en su majada y solo el 8,6 \% inyectaba complejos minerales-vitamínicos, una o dos veces al año a toda la majada en forma rutinaria, mientras que en otras majadas solo se trataba con estos complejos a los animales en mal estado.

Tabla 2: Producción de leche individual y total por cabra, porcentaje de cabritos señalados sobre cabras servidas y tasa de mortalidad perinatal, de acuerdo a si estacionan o no el servicio.

\begin{tabular}{|c|c|c|c|c|}
\hline $\begin{array}{l}\text { Estaciona } \\
\text { servicio }\end{array}$ & $\begin{array}{l}\text { Leche/diaria } \\
\text { (litros) }\end{array}$ & $\begin{array}{l}\text { Leche total } \\
\text { (litros) }\end{array}$ & $\begin{array}{l}\text { Cabritos señalados } \\
\text { /Cabras servidas }\end{array}$ & $\begin{array}{l}\text { Mortalidad } \\
\text { Perinatal }\end{array}$ \\
\hline Si $\quad(n=18)$ & 0,70 & 119,8 & $84,6 \%$ & $11,0 \%$ \\
\hline No $(n=17)$ & 0,57 & 82,7 & $71,9 \%$ & $22,1 \%$ \\
\hline
\end{tabular}


El 65,7 \% de los productores desparasitaba contra nematodes gastrointestinales en forma rutinaria a toda la majada, mayormente en otoño (52,9\%) o primavera $(41,2 \%)$ antes de subir a los cerros o al regresar de los mismos previo a la parición. Además, un 36,4\% de los productores que desparasitan llegan a tratar sus majadas de 3 a 4 veces repartidas en el año. De estos tratamientos en el 60, 31,4 y 8,6 \% de los casos se usa respectivamente ivermectina, closantel y albendazole.

Un 48\% trataba contra Fasciola hepatica mayormente con closantel en otoño.

Además, en el $30 \%$ de las UPF se trataba a los cabritos rutinariamente mediante piojicidas en base a piretroides aplicados por derrame dorsal o en menor medida con ivermectina y en el resto solo se trataba a los que se los veía muy parasitados.

$\mathrm{Al}$ analizar las variables productivas de acuerdo a si la majada era desparasitada rutinariamente, la tendencia revela datos más eficientes en aquellas UPF donde se desparasitaba, mostrando en el caso de la producción láctea individual diferencias $(\mathrm{p}<0,07)$ importantes (Tabla 3). En cuanto a la producción de leche total por cabra se halló una probabilidad 2,20 veces mayor de obtener más de $100 \mathrm{l}$ de leche total desparasitando a la majada $(\mathrm{OR}=2,20)$. También existe una tendencia en aquellas UPF que desparasitan y estacionan servicio a obtener más $(<0,08)$ litros totales $(131,9 \pm 62)$ sobre aquellas que solo estacionan $(75,0 \pm 27)$ o que solo desparasitan $(74,0 \pm 32)$ o que no estacionan o no desparasitan (88,5 \pm 61$)$.

Al analizar el manejo sanitario de la majada, se puede observar la falta total de uso de vacunas preventivas, o el exceso de uso de antiparasitarios en una región árida donde la incidencia de los nematodes gastrointestinales es baja y debería priorizarse el diagnóstico antes de desparasitar. Sin embargo, los tratamientos, principalmente en el otoño previo al parto podrían incidir sobre la producción de leche (Suarez et al., 2017c), ya que eliminarían las cargas de nematodes gastrointestinales preexistentes acumuladas durante el verano y que al inicio del otoño pueden alcanzar niveles moderados (Suarez et al., 2018) y fundamentalmente eliminarían las cargas de Fasciola hepatica (Suarez, datos no publicados)

Las escasas prácticas sanitarias registradas señalan el poco conocimiento sobre su importancia tanto productiva como para la salud de las familias, tal cual fue registrado también en otras encuestas a pequeños productores de diferentes regiones de Argentina (Mancebo et al., 2011; Suarez, et al., 2015; 2016).

Tabla 3: Producción de leche individual y total por lactancia por cabra, porcentaje de cabritos señalados sobre cabras servidas y tasa de mortalidad perinatal, de acuerdo a si desparasitaban rutinariamente o no a la majada.

\begin{tabular}{|c|c|c|c|c|}
\hline $\begin{array}{c}\text { Desparasita } \\
\text { anualmente }\end{array}$ & $\begin{array}{l}\text { Leche/diaria } \\
\text { (litros) }\end{array}$ & $\begin{array}{l}\text { Leche total } \\
\text { (litros) }\end{array}$ & $\begin{array}{l}\text { Cabritos señalados } \\
\text { /Cabras servidas }\end{array}$ & Mortalidad Perinatal \\
\hline Si $(n=23)$ & 0,68 & 109,9 & $86,8 \%$ & $15,0 \%$ \\
\hline No $(n=12)$ & 0,49 & 85,3 & $66,9 \%$ & $18,2 \%$ \\
\hline
\end{tabular}




\section{CONCLUSIONES}

Los resultados muestran por donde transitarían las limitantes de la cría caprina asociada a los sistemas de la agricultura familiar de la región estudiada, considerando siempre los sesgos de la presente encuesta en cuanto a la elección de las UPF. Dejando de lado los problemas ligados a la aridez del ambiente y los escasos recursos de las familias productoras, algunas de las limitantes en cuanto a la producción de leche y de carne podrían sin costos adicionales ser reducidas. En principio los resultados señalan la importancia de estacionar el servicio y la posibilidad consecuente de un mejor manejo forrajero acorde a los requerimientos fisiológicos de las cabras, a partir del uso apropiado de la alfalfa y el pastoreo en los cerros y rastrojos. También el manejo de los servicios posibilitaría un mejor control de las pariciones y una disminución de la mortalidad perinatal. Por otro lado, los resultados muestran que se debería profundizar el estudio del manejo sanitario más apropiado para eficientizar la cría de los caprinos, como buscar alternativas dentro de las familias para poder incrementar el tiempo neto de pastoreo diario de las cabras y así mejorar sus requerimientos nutricionales.

Finalmente, a pesar de que el muestreo solo abarcó el municipio de Payogasta, estudios puntuales previos en otras regiones de los Valles Calchaquíes indicarían que estos resultados podrían extrapolarse a otras zonas de estos valles sirviendo de base para futuras investigaciones.

\section{AGRADECIMIENTOS}

Los autores quieren expresar su agradecimiento a las familias por su gran predisposición y participación en la encuesta a partir de sus opiniones. También un reconocimiento al Municipio de Payogasta por contribuir con el financiamiento y con la ejecución del presente trabajo.

\section{BIBLIOGRAFÍA}

1.- BEDOTTI, D. O.; GÓMEZ CASTRO, A. G.; GARCÍA MARTÍNEZ, A.; SÁNCHEZ RODRÍGUEZ, M.; PEREA MUÑOZ, J. Y RODRÍGUEZ ESTÉVEZ, V. 2007. Estructura productiva de las Explotaciones Caprinas del Oeste Pampeano (Argentina). Archivos de Zootecnia 56 (213): 91-94.

2.- CHAVEZ, M.S.; PEROTTI, L.; CORTEZ, S.H.; SALDAÑO, R.; OROSCO, S.; SANCHEZ, V.; DÍAZ, L. Y CANDOTTI, J.J. 2011. Calidad de leche caprina de dos zonas agroecológicas del Noroeste Argentino (NOA), valles áridos y quebradas y valle templado. Resúmenes del Congreso de la Asociación Argentina de Producción Animal, TPP/SP 95.

3.- CONTRERAS, M.C.; AUHAD, L.; RENOLFI, R.; RUIZ S.; MARTÍNEZ, M.; PIEDRASANTA, R.; ORELLANA, P.; LAZARTE, M. Y PISANO, P. 2016. Caracterización del manejo de la majada caprina en el suroeste Santiagueño. Serie de informes técnicos, INTA Ediciones, C. Divulgación, $\mathrm{N}^{\circ}$ 92: $23 \mathrm{p}$.

4.- DAYENOFF, P. Y CARRIZZO, H. 1993. Aproximación a la problemática de la producción caprina en los Llanos de La Rioja. Jornadas de Producción Caprina de Río Cuarto. 2 y 3 de septiembre, pp 14-19. 
Producción de caprinos en los Valles Calchaquíes

5.- FERNÁNDEZ, J.L.; RABASA, A.E.; HERNÁNDEZ, M.E.; HOLGADO, F.D.; SALDAÑO, S.A. Y JORRAT, J.J. 2007 Producción de leche de tres biotipos caprinos en la Provincia de Tucumán Resúmenes Revista Argentina de Producción Animal, Vol 27. Supl.I.

6.- FERNÁNDEZ, J.L.; HOLGADO, F.D.; HERNÁNDEZ, M.E.; SOLALIGUE, P.B. Y SALINAS, C. 2014. Caracterización morfológica del caprino Criollo del NOA I: Medidas morfométricas e índices corporales. Rev. Agron. Noroeste Argent., UNT, 34 (2): 107-110.

7.- GUTMAN, G.; ITURREGUI, M.E. Y FILADORO, A. 2004. Propuestas para la formulación de políticas para el desarrollo de tramas productivas regionales: El caso de la lechería caprina en Argentina. Informe CEPAL, 94 p.

8.- MANCEBO, O.A.; RUSSO, A.M.; GIMÉNEZ, J.N.; GAIT, J.J. Y MONZÓN, C.M. 2011. Enfermedades más frecuentes en caprinos de la provincia de Formosa (Argentina). Veterinaria Argentina, Vol. XXVIII, 274: 1-16.

9.- MANZANAL, M. 1987. Pobreza y marginalidad en el agro argentino. La producción agrícola y su comercialización en Cachi, Salta. Cuadernos del CEUR, Buenos Aires, Argentina 20, ISSN 0326-1417.

10.- MARTÍNEZ G.M. Y SUAREZ, V.H. 2019. Lechería Caprina: producción, manejo, sanidad, calidad de leche y productos. 1ra Ed. INTA Ediciones, Colección Investigación, desarrollo e innovación. 167 p. https://inta. gob.ar/documentos/lecheria-caprina-produccion-manejo-sanidad-calidad-de-leche

11.- PAZ, R.G., TOGO, J., USANDIVARAS, P., CASTEL, J.M. Y MENA, Y. 2005. Análisis de la diversidad en los sistemas lecheros caprinos y evaluación de los parámetros productivos en la principal cuenca lechera de Argentina. Livestock Research for Rural Development, Vol. 17, Art..8. February 18; http:// www.cipav.org.co/lrrd/ lrrd17/1/paz17008. htm [fecha de consulta: 1 octubre 2019].
12.- PAZ, R.G.; TOGO, J.A. Y LÓPEZ, C. 2007. Evaluación de parámetros de producción de leche en caprinos Santiago del Estero, Argentina) Revista Científica, FCV-LUZ / Vol. XVII, No 2: $161-165$.

13.- POLI, M.A.; ROLDÁN, D.L.; SUÁREZ, C.; FERNÁNDEZ, J.L.; SALDAÑO, S.A.; HOLGADO, F.D. Y RABASA, A.E. 2005. Caprinos criollos en Argentina: avances en la caracterización y evaluación productiva. Agrociencia Vol. IX, 1 y 2: 479-484.

14.- RIGALT, F.; HERRERA, V.; GÓMEZ, R. Y PIVOTTO, R. 1993.Sistemas de producción caprina en la provincia de Catamarca, Argentina. En; Iñiguez, L y Tejada E.(eds). Memorias de un taller sobre metodologías de la investigación. Tarija, Bolivia. 16-21 de Agosto., pp: 183-200

15.- RIAN 2010. Existencias ganaderas caprinas 2009-2010. Red de Información Agropecuaria Nacional, INTA RIAN, rian.inta.gov. ar/ [fecha de consulta: 1 septiembre 2019].

16.- SUÁREZ, V.H.; ROSETTO C.B.; GAIDO A.B.; SALATIN, A.O; BERTONI, E.A.; DODERO, A.M.; VIÑABAL, A.E.; PINTO, G.; BRIHUEGA; B., ROMERA S.A. Y MAIDANA S. 2015. Prácticas de manejo y presencia de enfermedades en majadas caprinas de la región del chaco salteño. Veterinaria Argentina, www.veterinariargentina.com, No 332 Diciembre, 2015 [fecha de consulta: 6 septiembre 2019].

17.- SUÁREZ, V.H.; DODERO, A.M.; NIEVAS, J.D.; MARTÍNEZ G.M.; BERTONI, E.A.; SALATIN, A.O.; VIÑABAL, A.E.; GROSSBERGER, G.; BRIHUEGA, B.; ROMERA S.A. Y PINTO G., 2016. Presencia de enfermedades en majadas caprinas de las quebradas áridas de Jujuy y Salta . Vet. Argentina. XXXIII, 342 www.veterinariargentina.com, octubre 2016, ISSN 1852317XSS [fecha de consulta: 23 agosto 2019]. 
18.- SUÁREZ, V.H.; MARTÍNEZ G.M.; 20.- SUAREZ, V.H.; MARTÍNEZ, G.M.; NIEVAS, J.D. Y QUIROGA ROGER. J. 2017a. Prácticas de manejo y producción en sistemas familiares de cría caprina en las quebradas áridas de Jujuy y Salta. Revista de Investigaciones Agropecuarias (INTA), 43 (2): 186-194.

19.- SUÁREZ, V.H.; DODERO, A.M.; ALMUDEVAR F.M.; BERTONI, E.A.; SALATIN, A.O; VIÑABAL, A.E.; SALDAÑO, R.; MARTÍNEZ, G.M.; MICHELOUD, J.F.; FIORENTINO, MA.; BRIHUEGA, B. Y ROMERA S.A. 2017b. Presencia de enfermedades y prácticas de manejo en majadas caprinas de los valles templados del noroeste Argentina. Veterinaria Argentina. XXXIV, 356 www.veterinariargentina.com, diciembre 2017, ISSN 1852-317X [fecha de consulta: 7 septiembre 2019]. VIÑABAL, A.E. Y ALFARO, J.R. 2017c. Epidemiology and effect of gastrointestinal nematodes on dairy goats in Argentina. Onderstepoort J Vet Res 84, 1: a1240. https://doi.org/10.4102/ojvr.v84i1.1240

21.- SUAREZ, V.H.; ECHAZÚ, F.; QUIROGA ROGER, J.A. Y VIÑABAL, A.E. 2018. Parásitos internos de caprinos y ovinos en las regiones de quebradas áridas y la puna de Jujuy (Argentina). Rev. med. vet. (B. Aires), 99 (2) $112-116$.

22.- THURSFIELD, M. 1990. Veterinary Epidemiology. Editorial Acribia S.A., Zaragoza., 339 pp. 